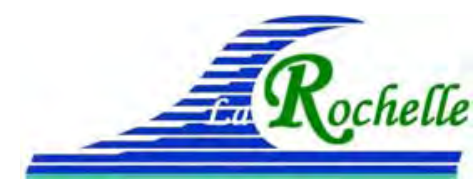

XVèmes Journées Nationales Génie Côtier - Génie Civil

La Rochelle, 29 au 31 mai 2018

DOI:10.5150/jngcgc.2018.101

(c) Editions Paralia CFL

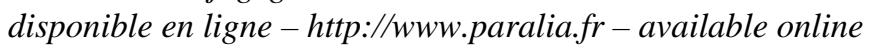

\title{
Observer les états de mer par télédétection: nouvelles techniques et applications, nouveaux satellites
}

\author{
Fabrice ARDHUIN ${ }^{1}$
}

1. UMR LOPS, Université de Brest, CNRS, Ifremer, IRD, IUEM, Rue Dumont d'Urville, 29280 Plouzané, France.ardhuin@ifremer.fr

\section{Résumé :}

La connaissance des états de mer s'appuie aujourd'hui largement la modélisation numérique, en particulier pour les applications de génie côtier. Ces modèles sont très partiellement validés pour leur contenu spectral en quelques points de mesure, par exemple le réseau CANDHIS ne compte qu'une vingtaine de houlographes. Ces observations ne permettent pas de valider les variations spatiales de l'état de la mer. Pour cela, la télédétection offre de nombreuses opportunités. En particulier les altimètres donnent des mesures fiables de la hauteur significative depuis 1993. Ces données sont indispensables pour la validation des modèles numériques, et peuvent révéler des détails intéressant, en particulier l'effet des courants sur les vagues. L'arrivée de nouvelles méthodes de traitement ainsi que de nouveaux altimètres utilisant un mode Doppler permet dès aujourd'hui de réduire considérablement le bruit de mesure et d'accéder à des échelles plus fines. Par ailleurs, les mesures spectrales se multiplient avec différentes techniques optiques et radar. Il convient en particulier de souligner le prochain lancement du China-France Ocean SATellite (CFOSAT) qui embarque un radar à vagues d'un genre nouveau, et le projet de satellite SKIM pour la mesure conjointe des courants et des vagues. Ces nouvelles techniques permettent de nouvelles analyses des états de mer, et une combinaison plus efficace entre modèle et observation.

\section{Mots-clés :}

Etats de mer, Télédétection, CFOSAT, Sentinel, SKIM, Modélisation, Validation.

\section{Introduction : l'altimétrie d'hier à aujourd'hui}

\subsection{Altimétrie classique par mesure du temps d'aller-retour}

Cela fait 25 ans que des satellites mesurent la hauteur significative des vagues. Ainsi, l'altimétrie radar est devenue la première source de données pour la climatologie des états de mer, comme illustré en figure 1. La plupart des missions spatiales ont utilisé la bande radar $\mathrm{Ku}(2,4 \mathrm{~cm}$ de longueur d'onde), avec une empreinte au sol du faisceau radar de l'ordre de $7 \mathrm{~km}$ de diamètre. Du fait de la faible revisite temporelle et de l'espacement entre les traces au sol, ces données sont relativement peu utilisées directement en génie côtier. En effet, il est possible de n'avoir aucune mesure dans une tempête donnée. Par contre, les altimètres sont indispensables pour calibrer et valider les 


\section{Lecture spéciale}

modèles numériques dans une grande variété de conditions. En particulier, l'altimétrie a démontré sa robustesse pour des hauteurs significatives comprises entre 0,8 et $20,1 \mathrm{~m}$ (HANAFIN et al., 2012).
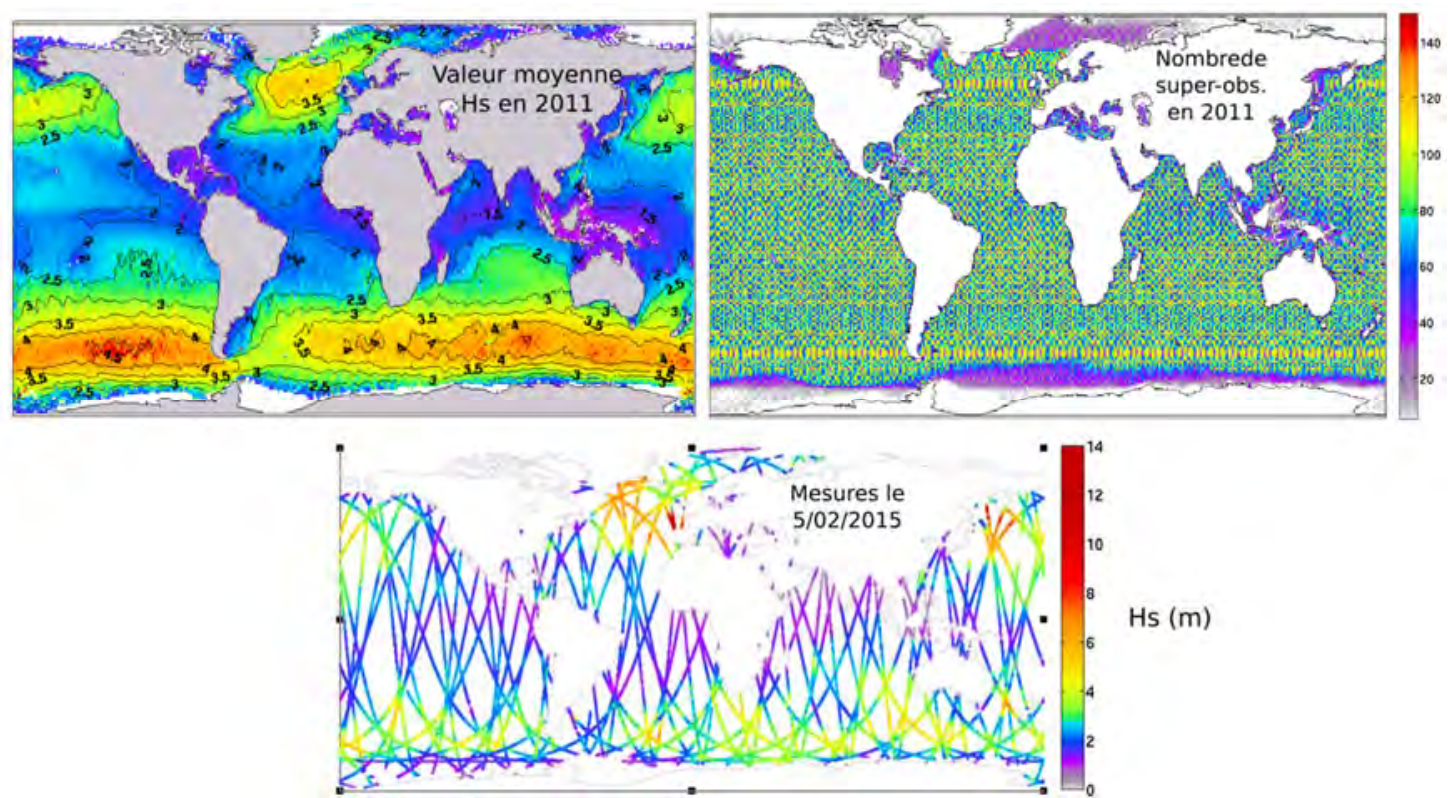

Figure 1. Illustration des mesures altimétriques : hauteur moyenne des vagues sur l'année 2011, nombre de "super-observations" (moyennes sur 1 degré de latitude) par carreau de $1^{\circ}$ par $1^{\circ}$ en une année, et exemple de couverture journalière le 5 février 2015, combinant les mesures de Jason 2, SARAL/Altika et Cryosat 2.

Les missions altimétriques ont été optimisées pour une mesure de la signature des grands courants et des tourbillons océaniques (DUCET et al., 2000), avec deux types d'orbites : une orbite "de référence" avec un passage tous les 10 jours et une latitute maximale de $66^{\circ}$, et une orbite plus inclinée avec une période de l'ordre de 35 jours et une latitude maximale autour de $82^{\circ}$. La première orbite a été occupée par TopexPoseidon (TP) puis Jason, Jason-2 et maintenant Jason-3 qui sera suivi par Sentinel-6 (en rouge sur la figure 1). Pour la second orbite il y a eu quelques variations : Envisat puis SARAL/AltiKa ont suivi exactement la même orbite (en jaune sur la figure 2), alors que ERS1, ERS2 et maintenant Sentinel 3 sont légèrement décalés. D'autres missions spatiales comme Geosat Follow On et HY2 complètent cette couverture. Un panorama général des données disponibles est donné par la figure 3. 


\section{$X V^{\text {èmes }}$ Journées Nationales Génie Côtier - Génie Civil \\ La Rochelle, 29 au 31 mai 2018}

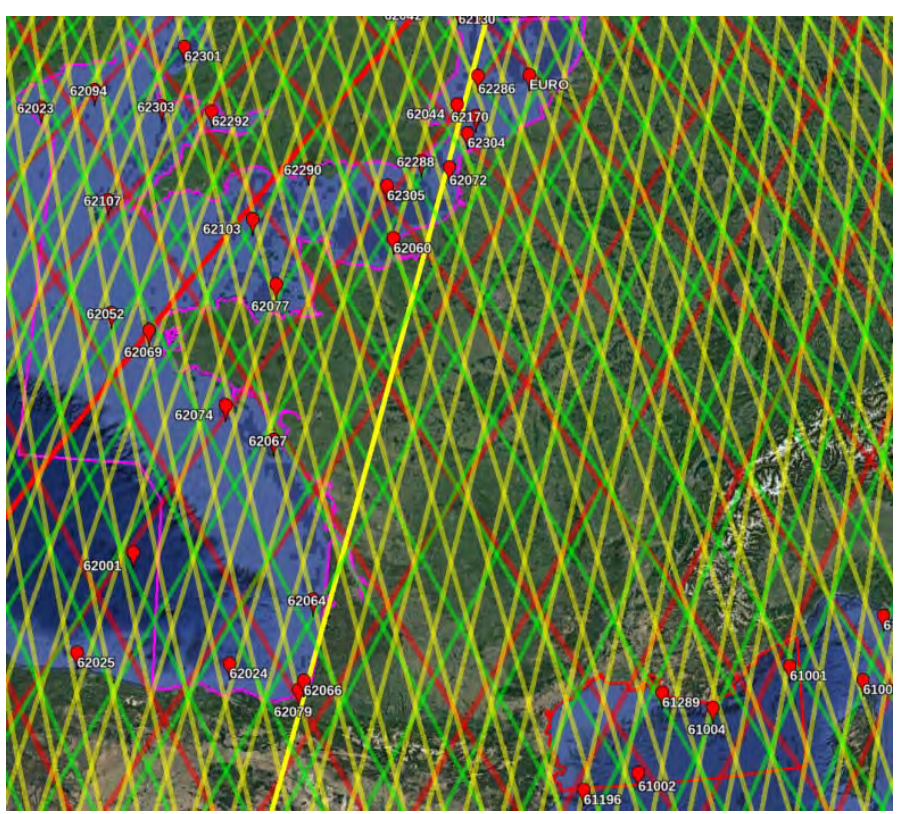

Figure 2. Couverture des traces altimétriques de Topex/Poseidon-Jason1/2/3-Sentinel 6 (en rouge), GeoSat Follow On (vert), Envisat-SARAL/AltiKa (jaune). Les polygones violet et rouge correspondent aux domaines des modèles NORGASUG et MENORUG mis en œuvre dans le cadre de Prévimer/MARC (http://marc.ifremer.fr ) et HOMERE. Deux passes altimétriques particulièrement intéressants sont soulignés : une passe Jason 3 qui longe la côte du Finistère, et une passe SARAL/AltiKa qui longe la côte Aquitaine.

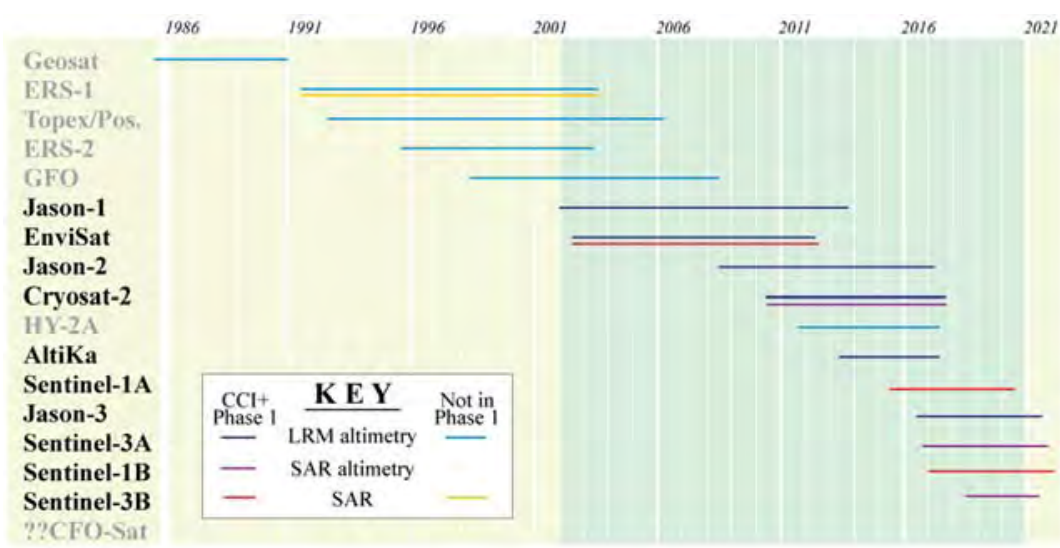

Figure 3. Historique des mesures d'état de mer par satellite utilisant des techniques altimétriques et imagerie SAR.

Outre la couverture spatiale et temporelle, qui est la première contrainte de l'observation par satellite, il y a eu une très forte évolution des techniques de mesure et de traitements. En effet, une des limitations pour les applications en zone côtière est le fort niveau de bruit associé aux méthodes d'estimation de la hauteur des vagues, et la contamination des échos de terre quand la trace au sol s'approche des côtes. Un exemple en figure 4 


\section{Lecture spéciale}

montre une trace descendante de Jason 3 sur laquelle deux traitements différents ont été appliqué. Le produit standard "GDR" utilise une méthode de Newton-Raphson tandis que le produit PEACHI est un produit expérimental qui utilise une méthode de NelderMead qui donne une estimation mois bruitée de la hauteur des vagues (ARDHUIN et al., 2017). Les deux traitements ont une variabilité très différente à des échelles inférieures à $1^{\circ}$ de latitude $(100 \mathrm{~km})$. En particulier la variabilité du produit classique à petite échelle est essentiellement du bruit qu'il vaut mieux filtrer. Les deux jeux de données donnent des valeurs localement fortes sur la côte nord du Finistère, jusqu'à $2,8 \mathrm{~m}$ à $48,7^{\circ} \mathrm{N}$, là où le modèle WAVEWATCH III mis en œuvre au LOPS (ROLAND \& ARDHUIN, 2014) sous-estime les valeurs observées avec seulement 2,2 m. Cela peut être lié à une sous-estimation de l'effet du courant dans ce cas de vent d'est.
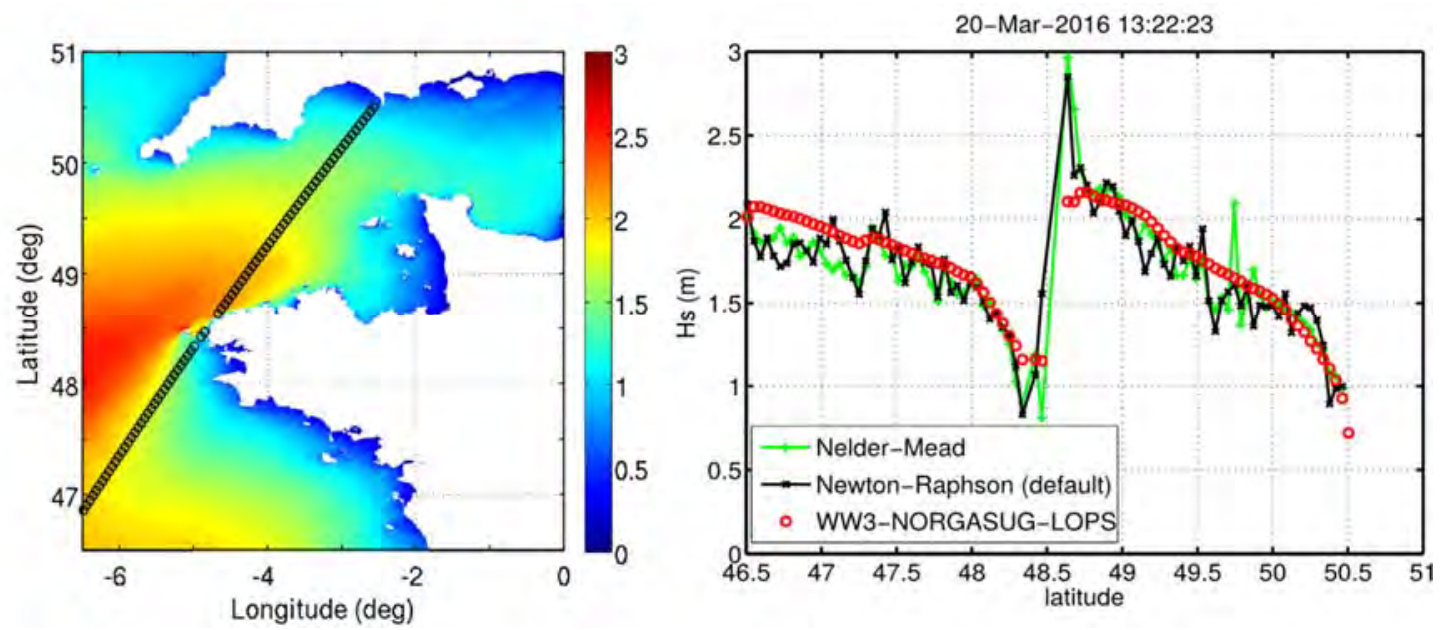

Figure 4. Exemple d'utilisation des données Jason 3, acquises le 20 mars 2016, pour la validation du modèle de vagues WAVEWATCH III mis en œuvre au LOPS. A gauche, carte de hauteurs de vagues avec les positions des mesures Jason 3 toutes les 1 s. A droite, comparaison entre modèle et observations, avec deux traitements différents des données altimétriques.

D'autres méthodes de traitement ont été développées pour les régions côtières et permettent aussi une réduction du bruit (PASSARO et al., 2015), qui peuvent aussi se combiner avec un débruitage a posteriori (QUILFEN et al., 2018).

Ainsi les altimètres peuvent être des outils précieux pour la mesure des gradients de l'état de mer, en particulier avec une utilisation de méthodes de traitement avancée. On notera, par exemple, que deux traces SARAL/AltiKa se croisent dans le Raz Blanchard, fournissant une excellente opportunité de suivi des états de mer complexes dans cette zone. 


\section{XVèmes Journées Nationales Génie Côtier - Génie Civil \\ La Rochelle, 29 au 31 mai 2018}

Une particularité de SARAL/AltiKa est l'utilisation de la bande radar Ka $(8 \mathrm{~mm}$ de longueur d'onde) et d'une plus grande largeur de bande, donnant une meilleure résolution en hauteur et un bruit plus faible (SEPULVEDA et al., 2015). La bande Ka a aussi été retenue pour le projet de mission spatiale SKIM, actuellement à l'étude par l'Agence Spatiale Européenne (ARDHUIN et al., 2018).

\subsection{Altimétrie Doppler}

Une évolution technique très importante a été testée sur Cryosat- 2 avec un traitement Doppler qui permet de d'augmenter fortement la résolution le long de la trace au sol : en plus du temps d'aller-retour des impulsions radar, le traitement Doppler analyse le décalage en fréquence des différents échos et permet de séparer les échos venant de l'avant (dans la direction de vol) de ceux venant de l'arrière. Un traitement SAR nonfocalisé permet ainsi d'arriver à une résolution de $300 \mathrm{~m}$, au lieu des $7 \mathrm{~km}$ de la tache au sol, et cette résolution peut aussi être exploitée pour améliorer la précision de la mesure (GARCIA et al., 2014). Cette technique est désormais utilisée sur Sentinel 3 et sera mise en œuvre sur Sentinel 6 et SKIM.

\section{Imagerie et mesure spectrale}

\subsection{Radars à ouverture synthétique (SARs)}

Avec les premiers essais de SEASAT en 1978, et le lancement de ERS1 en 1991, ne nombreux efforts ont été faits pour interpréter les images radar à haute résolution. En particulier les SARs de ERS1, ERS2, Envisat et Sentinel 1 opèrent sur l'océan en "mode vagues", qui permet un suivi des houles longues à travers les océans. Ces données ont permis une mesure de la dissipation de la houle et une nette amélioration des modèles numériques (ARDHUIN et al., 2009). En zone côtière, l'imagerie SAR est aussi capable de founir une cartographie détaillée des vagues, avec des limitations liées au principe de mesure. En effet, la haute résolution est perdue par l'effet de floutage associé aux vitesses orbitales des vagues qui contribuent au décalage Doppler des échos océaniques et la position des pixels sur l'image SAR. Le résultat est que dès que le vent est un peu fort, seules les vagues se propageant dans la direction de visée sont effectivement vues. Cette limitation fait que les SARs sont plutôt performants pour les houles et les vents faibles à modérés comme c'est le cas en figure 5. 


\section{Lecture spéciale}
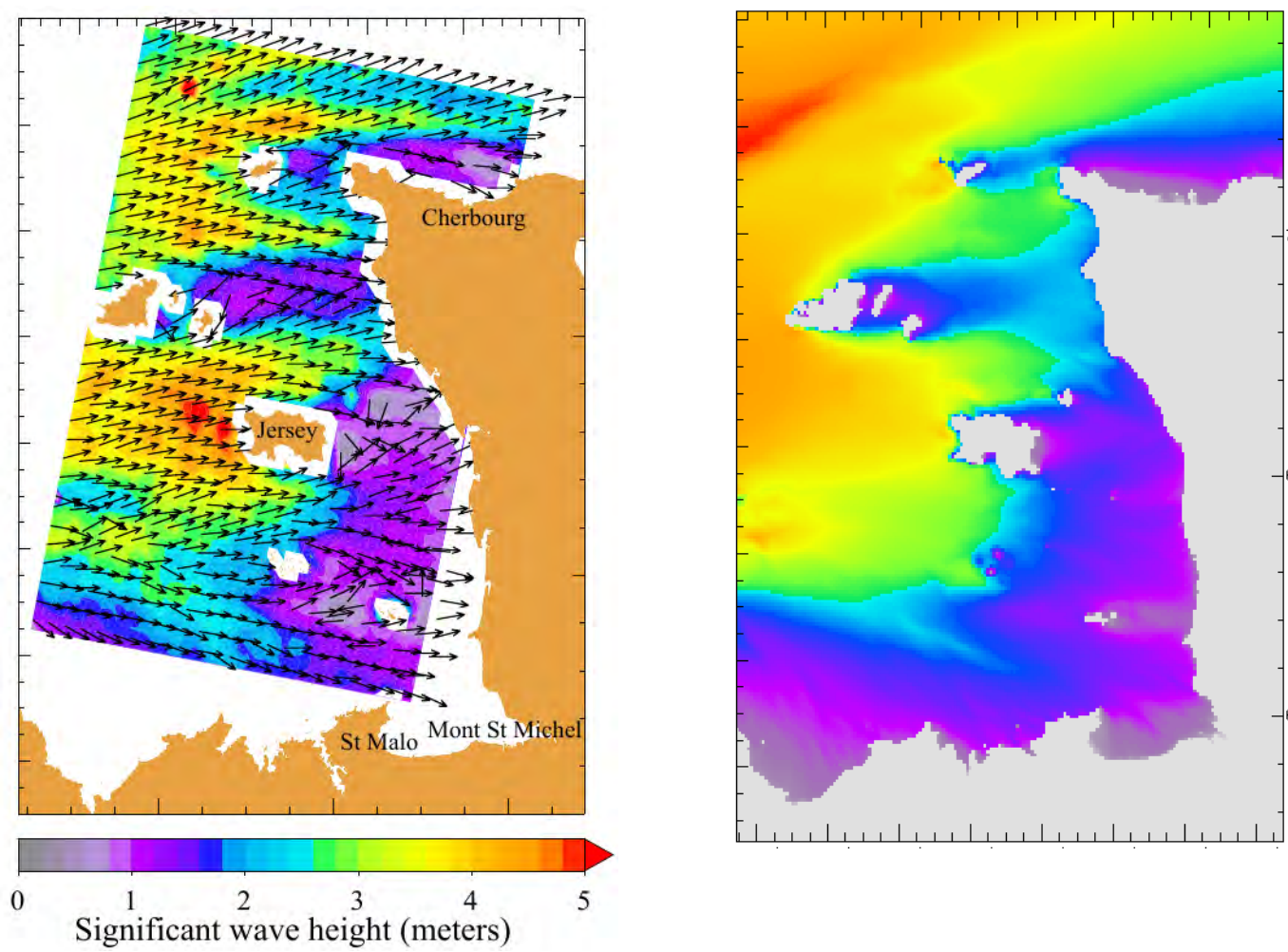

Figure 5. A gauche, exemple de carte de hauteurs de vagues et directions moyennes estimées à partir du SAR de Envisat le 9 Mars 2003 à $10 h 22$ (COLLARD et al., 2005). A droite résultats du modèle numérique WW3-NORGASUG mis en œuvre par le LOPS (ROLAND \& ARDHUIN, 2014).

\subsection{Radar à vagues}

Afin d'éviter cette limitation, on peut éviter le recours à la sythèse d'ouverture et à la très haute résolution dans la direction azimuthale. Dans ce cas on ne garde qu'une haute résolution dans la direction de visée, la modulation de ce signal est reliée à des vagues se propageant dans la direction de visée, avec une moyenne sur la tache au sol du radar (quelques kilomètres) dans la direction transverse (azimuth). C'est le principe de l'instrument SWIM dont le concept a été imaginé par Jackson dans les années 1980. La première mise en œuvre sur un satellite se fera avec la mission CFOSAT, dont le lancement est prévu en 2018. La combinaison des différents azimuths de visée permet de former un spectre directionnel. L'ajout d'une mesure Doppler, comme proposé sur SKIM permet au passage de lever l'ambiguïté sur le sens de propagation.

\subsection{Imagerie optique}

Les capteurs spatiaux récents, en particuler Landsat 8 et Sentinel 2, combinent à la fois une haute résolution spatiale (de l'ordre de $10 \mathrm{~m}$ ) et une large fauchée, ce qui permet une vision des états de mer sur de grandes étendues. En l'absence de nuages et dans des 


\section{XVèmes Journées Nationales Génie Côtier - Génie Civil \\ La Rochelle, 29 au 31 mai 2018}

conditions d'éclairement solaire favorable, KUDRYAVTSEV et al. (2017) ont montré que le capteur de Sentinel 2 était particulièrement adapté à une mesure des vagues et des courants, exploitant la relation entre radiance de l'image et pente de la surface, et la différence de temps entre l'acquisition des différents canaux de couleur. En particulier les différentes couleurs sont acquises par Sentinel 2 avec un décalage temporel qui atteint $0.8 \mathrm{~s}$, on peut donc déterminer le sens de propagation et même la vitesse du courant en comparant la phase du cross-spectre entre canaux avec la relation de dispersion linéaire.

\section{Conclusion}

Toute une série de développements récents, depuis de nouveaux traitements, jusqu'à de nouveaux instruments ont largement renouvelé les capacités de mesure des états de mer depuis l'espace. En particulier les mesures de hauteur significative par altimétrie, qui constituent le seul jeu de données véritablement global, ont été l'objet de développements importants qui vont beaucoup réduire le bruit de mesure et améliorer la résolution spatiale des données. Un test exaustif des méthodes de traitement sera fait dans le cadre du projet "Sea State Climate Change Initiative" de l'Agence Spatiale Européenne. Une première mise à jour de la base de données "Globwave" devrait ainsi être produite dès 2018. Les mesures spectrales ont elles aussi fait l'objet de développement important. L'année 2018, avec le lancement de CFOSAT, sera certainement l'occasion d'un renouveau important.

\section{Références bibliographiques}

ARDHUIN F., CHAPRON B., COLLARD F. (2009). Observation of swell dissipation across oceans. Geophys. Res. Lett., 36, p. L06607. https://doi.org/10.1029/2008GL037030

ARDHUIN F., RASCLE N., CHAPRON B., GULA J., MOLEMAKER J., GILLE S. T., MENEMENLIS D., ROCHA C. (2017). Small scale currents have large effects on wind wave heights. J. Geophys. Res., Vol. 122, C6, pp 4500-4517. https://doi.org/10.1002/2016JC012413

ARDHUIN F., et al. (2018). Measuring currents, ice drift, and waves from space: the Sea surface KInematics Multiscale monitoring (SKIM) concept. Ocean Sci., https://doi.org/10.5194/os-2017-65

COLLARD F., ARDHUIN F., CHAPRON B. (2005). Extraction of coastal ocean wave fields from SAR images. IEEE J. Oceanic Eng., Vol. 30(3), pp 526-533.

DUCET N., TRAON P.Y.L., REVERDIN G. (2000). Global high-resolution mapping of ocean circulation from TOPEX/Poseidon and ERS-1 and -2. J. Geophys. Res., Vol. 105, pp 19477-19498.

GARCIA E.S., SANDWELL D.T. SMITH W.H. (2014). Retracking CryoSat-2, Envisat and Jason-1 radar altimetry waveforms for improved gravity field recovery. Geophysical Journal International, Vol. 196(3), pp 1402-1422. 


\section{Lecture spéciale}

HANAFIN et al. (2012). Phenomenal sea states and swell radiation: a comprehensive analysis of the 12-16 February 2011 North Atlantic storms. Bull. Amer. Meterol. Soc., Vol. 93, pp 1825-1832. https://doi.org/10.1175/BAMS-D-11-00128.1

HAUSER D., TISON C., AMIOT T., DELAYE L., CORCORA, N., CASTILlA P. (2017). SWIM: The first spaceborne wave scatterometer. IEEE Trans. on Geosci. and Remote Sensing, Vol. 55(5), pp 3000-3014.

KUDRYAVTSEV V., YUROVSKAYA M., CHAPRON B., COLLARD F., DONLON C. (2017). Sun glitter imagery of surface waves. part 1: Directional spectrum retrieval and validation. J. Geophys. Res., Vol. 122. https://doi.org/10.1002/2016JC012425

PASSARO M., FENOGLIO-MARC L., CIPOLINI P. (2015). Validation of significant wave height from improved satellite altimetry in the German Bight. IEEE Trans. on Geosci. and Remote Sensing, Vol. 53, pp 2146-2156.

QUILFEN Y., YUROVSKAYA M., CHAPRON B., ARDHUIN F. (2018). Storm waves sharpening in the agulhas current: satellite observations and modeling. Remote sensing of Environment, in press.

ROLAND A., ARDHUIN F. (2014). On the developments of spectral wave models: numerics and parameterizations for the coastal ocean. Ocean Dynamics, Vol. 64(6), pp 833-846. https://doi.org/10.1007/s10236-014-0711-z

SEPULVEDA H.H., QUEFFEULOU P., ARDHUIN F. (2015). Assessment of SARAL AltiKa wave height measurements relative to buoy, Jason-2 and Cryosat-2 data. Marine Geodesy, Vol. 38, S1, pp 449-465. https://doi.org/10.1080/01490419.2014.1000470 